\title{
Further evidence that genotypically closely related strains of Legionella pneumophila can express different serogroup specific antigens
}

\author{
T. G. HARRISON, N. A. SAUNDERS, A. HATHTHOTUWA, N. DOSHI and A. G. TAYLOR \\ PHLS Legionella Reference Unit, Division of Microbiological Reagents, Central Public Health Laboratory, \\ 61 Colindale Avenue, London NW9 5HT
}

\begin{abstract}
Summary. The relationship between serogroup and genotype of Legionella pneumophila strains was investigated by restriction fragment length polymorphism (RFLP) typing with a previously standardised method. Of the 51 RFLP types identified, 19 comprised strains of more than one serogroup. Several RFLP types included strains of five or more serogroups. To determine if sharing the same RFLP type indicates that strains are genotypically indistinguishable or merely that they are superficially similar, 31 strains were selected for further analysis with an extended range of restriction endonucleases and nucleic acid probes. In some cases, strains of a particular RFLP type were indistinguishable, while in others the restriction fragment patterns showed minor differences. It is possible that in the latter case the strains are diverging representatives of a parent clone. We conclude that analysis of restriction fragment patterns, either probed or unprobed, provides a more accurate measure of the ancestral relationship between strains than can be obtained with serological methods.
\end{abstract}

\section{Introduction}

In 1988, van Ketel $^{1}$ reported a cluster of Legionella pneumophila strains of different serogroups with similar restriction endonuclease digest analysis (REA) profiles and suggested that the genetic similarity between strains of different serogroups is sometimes closer than between strains of the same serogroup. However, he acknowledged that small differences in REA profiles are difficult to detect because of the multitude of fragments produced, and thus the degree of similarity between the strains indistinguishable by REA remains unclear.

We have reported previously the development and evaluation of a highly discriminatory and reproducible typing method for L. pneumophila serogroup 1 strains, based on the use of biotinylated cloned DNA probes for the analysis of restriction fragment length polymorphisms (RFLPs). ${ }^{2}$ By revealing only a small subset of restriction fragments this technique often allows even minor differences between REA profiles to be distinguished. Recently we demonstrated the usefulness of this technique for typing $L$. pneumophila strains of any serogroup. ${ }^{3}$ In the course of these latter studies we noted that the RFLP type showed little concordance with serogroup, since some strains from several different serogroups had the same RFLP pattern. Therefore, RFLP typing was used in the present study as a supplement to REA to allow a more

Received 10 July 1991 ; revised version accepted 1 Nov. 1991. detailed investigation of the relationship between genotype and serogroup.

\section{Materials and methods}

\section{Bacterial strains}

Strains of L. pneumophila serogroups 1-14 were submitted to the PHLS Legionella Reference Unit (LRU) for routine examination. They were isolated between 1979 and 1988 from sources in Europe, North America and Asia. Additional strains were obtained from the National Collection of Type Cultures, Colindale. The identity of all strains was confirmed from nutritional, biochemical and serological characteristics. ${ }^{4}$ The strains, which were stored on glass beads at $-70^{\circ} \mathrm{C}$, were recovered on to BCYE agar and incubated for $72 \mathrm{~h}$ at $37^{\circ} \mathrm{C}$ before the growth was harvested in saline and pelleted by centrifugation. Data relating to more than 500 strains of L. pneumophila were considered in this study, some of which have been published in detail elsewhere. ${ }^{3}$

Thirty-one strains comprising three groups (table I) were selected for detailed examination with the following range of restriction enzymes and nucleic acid probes:

Group (i): RFLP type 28 strains. This group comprised seven strains each of a different serogroup.

Group (ii): RFLP type 26 strains. This group comprised 12 strains of five serogroups, including 
Table I. Summary of the 31 strains selected for detailed analysis

\begin{tabular}{|c|c|c|c|c|c|c|}
\hline Group & Strain no. & $C$ or $E$ & Source & $\begin{array}{l}\text { Date of } \\
\text { isolation }\end{array}$ & $\begin{array}{l}\text { RFLP } \\
\text { type }\end{array}$ & Serogroup \\
\hline (i) & $\begin{array}{l}\text { ML68 } \\
\text { LC727A } \\
\text { LC232 } \\
\text { LC100 } \\
\text { LC1 } \\
\text { LC802 } \\
\text { NCTC12000 }\end{array}$ & $\begin{array}{l}\mathrm{C} \\
\mathrm{E} \\
\mathrm{C} \\
\mathrm{C} \\
\mathrm{E} \\
\mathrm{E} \\
\mathrm{C}\end{array}$ & $\begin{array}{l}\text { UK } \\
\text { UK } \\
\text { Spain } \\
\text { France } \\
\text { UK } \\
\text { Holland } \\
\text { Holland }\end{array}$ & $\begin{array}{r}04 / 85 \\
? / 88 \\
03 / 87 \\
10 / 81 \\
01 / 85 \\
? / 89 \\
01 / 80\end{array}$ & $\begin{array}{l}28 \\
28 \\
28 \\
28 \\
28 \\
28 \\
28\end{array}$ & $\begin{array}{r}1 \\
2 \\
3 \\
6 \\
8 \\
9 \\
10\end{array}$ \\
\hline \multirow[t]{4}{*}{ (ii) } & $\begin{array}{l}\text { ML54 } \\
\text { LC563 }\end{array}$ & $\begin{array}{l}\mathrm{C} \\
\mathrm{E}\end{array}$ & $\begin{array}{l}\text { France } \\
\text { Austria }\end{array}$ & $\begin{array}{l}? / 84 \\
? / 88\end{array}$ & $\begin{array}{l}26 \\
26\end{array}$ & $\begin{array}{l}1 \\
3\end{array}$ \\
\hline & $\left.\begin{array}{l}\text { LC362 } \\
\text { LC366 }\end{array}\right\}$ & $\begin{array}{l}\mathrm{E} \\
\mathrm{C}\end{array}$ & $\begin{array}{l}\text { USA } \\
\text { USA }\end{array}$ & $\begin{array}{l}? / ? \\
? / ?\end{array}$ & $\begin{array}{l}26 \\
26\end{array}$ & $\begin{array}{l}6 \\
6\end{array}$ \\
\hline & $\left.\begin{array}{l}\text { LRU57 } \\
\text { LC202 } \\
\text { LC443 } \\
\text { LC207 } \\
\text { LC441 } \\
\text { LC204 } \\
\text { LC442 }\end{array}\right\}$ & $\begin{array}{l}\mathrm{C} \\
\mathrm{C} \\
\mathrm{E} \\
\mathrm{E} \\
\mathrm{C} \\
\mathrm{E} \\
\mathrm{E}\end{array}$ & $\begin{array}{l}\text { UK } \\
\text { UK } \\
\text { UK } \\
\text { UK } \\
\text { UK } \\
\text { UK } \\
\text { UK }\end{array}$ & $\begin{array}{r}? / 87 \\
? / 87 \\
? / 87 \\
? / 87 \\
10 / 87 \\
? / 87 \\
10 / 87\end{array}$ & $\begin{array}{l}26 \\
26 \\
26 \\
26 \\
26 \\
26 \\
26\end{array}$ & $\begin{array}{r}6 \\
6 \\
6 \\
6 \\
12 \\
12 \\
12\end{array}$ \\
\hline & LC467 & $\mathrm{C}$ & UK & $11 / 87$ & 26 & 10 \\
\hline (iii) & $\left.\begin{array}{l}\text { LC390 } \\
\text { LC392 } \\
\text { LC393 } \\
\text { LC394 } \\
\text { LC396 } \\
\text { LC398 } \\
\text { LC395 } \\
\text { LC397 } \\
\text { LC384 } \\
\text { LC385 } \\
\text { LC389 } \\
\text { LC391 }\end{array}\right\}$ & $\begin{array}{l}\mathrm{C} \\
\mathrm{C} \\
\mathrm{C} \\
\mathrm{C} \\
\mathrm{E} \\
\mathrm{E} \\
\mathrm{E} \\
\mathrm{E} \\
\mathrm{C} \\
\mathrm{C} \\
\mathrm{C} \\
\mathrm{C}\end{array}$ & $\begin{array}{l}\text { Belgium } \\
\text { Belgium } \\
\text { Belgium } \\
\text { Belgium } \\
\text { Belgium } \\
\text { Belgium } \\
\text { Belgium } \\
\text { Belgium } \\
\text { Belgium } \\
\text { Belgium } \\
\text { Belgium } \\
\text { Belgium }\end{array}$ & $\begin{array}{l}07 / 83 \\
05 / 84 \\
03 / 84 \\
01 / 86 \\
07 / 84 \\
07 / 84 \\
07 / 84 \\
07 / 84 \\
08 / 81 \\
02 / 82 \\
07 / 83 \\
12 / 83\end{array}$ & $\begin{array}{l}28 \\
28 \\
28 \\
28 \\
15 \\
15 \\
15 \\
15 \\
15 \\
15 \\
15 \\
15\end{array}$ & $\begin{array}{r}6 \\
6 \\
6 \\
6 \\
6 \\
6 \\
10 \\
10 \\
10 \\
10 \\
10 \\
10\end{array}$ \\
\hline
\end{tabular}

$\mathrm{C}$, clinical isolate; $\mathrm{E}$, environmental isolate.

Strains bracketed together are epidemiologically associated as described in the text; LC389 and LC390 were both isolated from the same patient.

clinical and environmental strains representative of two outbreaks of Legionnaires' disease (LD), one pair from an outbreak in the USA (J. Barbaree, personal communication) and seven serogroup 6 and serogroup 12 strains from a renal unit-associated outbreak of LD. Some of these latter strains have been described elsewhere. $^{5}$

Group (iii): 12 strains, collected over a 3-year period, from an outbreak in a Belgian hospital. ${ }^{6}$ Clinical and environmental isolates of both serogroup 6 and serogroup 10 strains (including a dual infection) were included. Previous work had shown that the serogroup 10 strains (environmental and clinical) and environmental serogroup 6 strains were all RFLP type 15. However, the clinical serogroup 6 strains were RFLP type $28 .^{3}$

\section{Restriction endonuclease enzymes}

In preliminary experiments, a range of restriction enzymes was assessed for suitability for RFLP typing. The following enzymes were chosen for use in this study because they gave clear, simple RFLP patterns and could distinguish between test strains: NciI, AvaII, StyI, BanI and HaeIII (Anglian Biotech Ltd).

\section{Restriction endonuclease digest analysis (REA)}

DNA was extracted from bacterial pellets by the guanidine isothiocyanate method described previously. ${ }^{7}$ The isolated DNA was digested to completion according to the manufacturer's instructions. The restriction fragments were subjected to agarose gel electrophoresis (agarose $0.8 \%, 1 \mathrm{~V} / \mathrm{cm}, 16 \mathrm{~h}$ ) and, where appropriate, were stained with ethidium bromide $0.5 \mu \mathrm{g} / \mathrm{ml}$ for $30 \mathrm{~min}$.

\section{Restriction fragment length polymorphism (RFLP) typing}

RFLP typing was done as described by Saunders $e t$ $a l^{2}$ with the biotinylated probes $\lambda 20 / 21, \lambda 142$ and $\lambda$ LEG $1^{8}$ and the restriction endonucleases detailed above. The probes $\lambda 20 / 21$ and $\lambda$ LEG1 are $\lambda$ phage clones containing $L$. pneumophila chromosome fragments of unknown function and $\lambda 142$ is a clone containing a rRNA cistron from this species. The reproducibility of RFLP typing with probe $\lambda 20 / 21$ and enzyme NciI has been thoroughly evaluated previously. Banding patterns were analysed and strains allocated to an RFLP type as described previously. ${ }^{2}$ 
Table II. Summary of RFLP types comprising L. pneumophila strains of more than one serogroup

\begin{tabular}{|c|c|c|c|c|}
\hline Representative* & $\begin{array}{l}\text { Similar } \dagger \\
\text { strains }\end{array}$ & Source & Serogroup§ & RFLP type \| \\
\hline $\begin{array}{l}\text { ML56 } \\
\text { NCTC11986 }\end{array}$ & $\begin{array}{r}26 \\
1\end{array}$ & $\begin{array}{l}\text { UK (C) } \\
\text { USA (C) }\end{array}$ & $\begin{array}{l}1 \\
9\end{array}$ & $\begin{array}{l}1 \\
1\end{array}$ \\
\hline $\begin{array}{l}\text { LC423 } \\
\text { LC840 } \\
\text { LC608 } \\
\text { A247/81 } \\
\text { LC1156 }\end{array}$ & $\begin{array}{r}12 \\
2 \\
3 \\
2 \\
1\end{array}$ & $\begin{array}{l}\text { UK (C) } \\
\text { Cyprus (C) } \\
\text { UK (E) } \\
\text { UK (E) } \\
\text { UK (E) }\end{array}$ & $\begin{array}{l}1 \\
3 \\
5 \mathrm{D} \\
6 \\
12\end{array}$ & $\begin{array}{l}5 \\
5 \\
5 \\
5 \\
5\end{array}$ \\
\hline $\begin{array}{l}\text { LC409 } \\
\text { LC258 } \\
\text { LC419 } \\
\text { NCTC12181 }\end{array}$ & $\begin{array}{l}3 \\
1 \\
1 \\
1\end{array}$ & $\begin{array}{l}\text { UK (E) } \\
\text { Singapore (E) } \\
\text { Singapore (E) } \\
\text { USA (C) }\end{array}$ & $\begin{array}{r}1 \\
7 \\
8 \\
13\end{array}$ & $\begin{array}{l}7 \\
7 \\
7 \\
7\end{array}$ \\
\hline $\begin{array}{l}\text { ML70 } \\
\text { LC229 }\end{array}$ & $\begin{array}{l}3 \\
1\end{array}$ & $\begin{array}{l}\text { UK (C) } \\
\text { UK (C) }\end{array}$ & $\begin{array}{l}1 \\
8\end{array}$ & $\begin{array}{l}10 \\
10\end{array}$ \\
\hline $\begin{array}{l}\text { A32/81 } \\
\text { A89/81 } \\
\text { LC811 }\end{array}$ & $\begin{array}{l}1 \\
2 \\
1\end{array}$ & $\begin{array}{l}\text { UK (E) } \\
\text { Australia (E) } \\
\text { UK (E) }\end{array}$ & $\begin{array}{r}1 \\
6 \\
10\end{array}$ & $\begin{array}{l}11 \\
11 \\
11\end{array}$ \\
\hline $\begin{array}{l}\text { LC19 } \\
\text { NCTC11287 } \\
\text { LC812 }\end{array}$ & $\begin{array}{l}2 \\
2 \\
1\end{array}$ & $\begin{array}{l}\text { France (C) } \\
\text { UK (E) } \\
\text { UK (E) }\end{array}$ & $\begin{array}{l}1 \\
6 \\
8\end{array}$ & $\begin{array}{l}13 \\
13 \\
13\end{array}$ \\
\hline $\begin{array}{l}\text { A218/80 } \\
\text { LC796 } \\
\text { LC384 }\end{array}$ & $\begin{array}{l}1 \\
4 \\
6\end{array}$ & $\begin{array}{l}\text { UK (E) } \\
\text { UK (E) } \\
\text { Belgium (C) }\end{array}$ & $\begin{array}{r}1 \\
6 \\
10\end{array}$ & $\begin{array}{l}15 \\
15 \\
15\end{array}$ \\
\hline $\begin{array}{l}\text { A } 176 / 79 \\
\text { NCTC11984 }\end{array}$ & $\begin{array}{l}1 \\
1\end{array}$ & $\begin{array}{l}\text { USA (C) } \\
\text { USA (E) }\end{array}$ & $\begin{array}{l}1 \\
7\end{array}$ & $\begin{array}{l}16 \\
16\end{array}$ \\
\hline $\begin{array}{l}\text { LC372 } \\
\text { NCTC12179 }\end{array}$ & $\begin{array}{l}2 \\
1\end{array}$ & $\begin{array}{l}\text { UK (C) } \\
\text { USA (C) }\end{array}$ & $\begin{array}{r}1 \\
11\end{array}$ & $\begin{array}{l}19 \\
19\end{array}$ \\
\hline $\begin{array}{l}\text { ML60 } \\
\text { LC543 } \\
\text { LC569 }\end{array}$ & $\begin{array}{r}3 \\
1 \\
14\end{array}$ & $\begin{array}{l}\text { UK (C) } \\
\text { UK (E) } \\
\text { UK (C/E) }\end{array}$ & $\begin{array}{l}1 \\
6 \\
8\end{array}$ & $\begin{array}{l}25 \\
25 \\
25\end{array}$ \\
\hline $\begin{array}{l}\text { ML54 } \\
\text { LC563 } \\
\text { NCTC11406 } \\
\text { LC467 } \\
\text { LC204 }\end{array}$ & $\begin{array}{r}8 \\
2 \\
26 \\
1 \\
12\end{array}$ & $\begin{array}{l}\text { UK (C) } \\
\text { Austria (E) } \\
\text { USA (C) } \\
\text { UK (E) } \\
\text { UK (C) }\end{array}$ & $\begin{array}{r}1 \\
3 \\
6 \\
10 \\
12\end{array}$ & $\begin{array}{l}26 \\
26 \\
26 \\
26 \\
26\end{array}$ \\
\hline $\begin{array}{l}\text { ML68 } \\
\text { LC727A } \\
\text { LC232 } \\
\text { LC100 } \\
\text { LC1 } \\
\text { LC802 } \\
\text { NCTC12000 }\end{array}$ & $\begin{array}{r}4 \\
3 \\
8 \\
11 \\
3 \\
3 \\
1\end{array}$ & $\begin{array}{l}\text { UK (C) } \\
\text { UK (E) } \\
\text { Spain (C) } \\
\text { France (C) } \\
\text { UK (E) } \\
\text { Holland (E) } \\
\text { Holland (C) }\end{array}$ & $\begin{array}{r}1 \\
2 \\
3 \\
6 \\
8 \\
9 \\
10\end{array}$ & $\begin{array}{l}28 \\
28 \\
28 \\
28 \\
28 \\
28 \\
28\end{array}$ \\
\hline $\begin{array}{l}\text { LC564 } \\
\text { LC767 } \\
\text { ML87 } \\
\text { LC831 }\end{array}$ & $\begin{array}{l}1 \\
1 \\
1 \\
1\end{array}$ & $\begin{array}{l}\text { Austria (E) } \\
\text { UK (E) } \\
\text { Hong Kong (E) } \\
\text { UK (C) }\end{array}$ & $\begin{array}{l}1 \\
6 \\
1 \\
8\end{array}$ & $\begin{array}{l}29 \\
29 \\
31 \\
31\end{array}$ \\
\hline $\begin{array}{l}\text { LC3 } \\
\text { LC223 } \\
\text { LC227 } \\
\text { LC572 }\end{array}$ & $\begin{array}{l}1 \\
5 \\
2 \\
1\end{array}$ & $\begin{array}{l}\text { UK (E) } \\
\text { UK (E) } \\
\text { UK (E) } \\
\text { UK (E) }\end{array}$ & $\begin{array}{r}2 \\
3 \\
6 \\
14\end{array}$ & $\begin{array}{l}34 \\
34 \\
34 \\
34\end{array}$ \\
\hline $\begin{array}{l}\text { LC638 } \\
\text { NCTC11232 }\end{array}$ & $\begin{array}{l}1 \\
1\end{array}$ & $\begin{array}{l}\text { UK (E) } \\
\text { USA (E) }\end{array}$ & $\begin{array}{l}1 \\
3\end{array}$ & $\begin{array}{l}37 \\
37\end{array}$ \\
\hline $\begin{array}{l}\text { LRU71 } \\
\text { LC570 }\end{array}$ & $\begin{array}{l}1 \\
1\end{array}$ & $\begin{array}{l}\text { UK (E) } \\
\text { UK (E) }\end{array}$ & $\begin{array}{r}3 \\
14\end{array}$ & $\begin{array}{l}40 \\
40\end{array}$ \\
\hline $\begin{array}{l}\text { LC574 } \\
\text { LC560 } \\
\text { LC573 } \\
\text { LC575 }\end{array}$ & $\begin{array}{l}1 \\
1 \\
1 \\
1\end{array}$ & $\begin{array}{l}\text { UK (E) } \\
\text { Austria (E) } \\
\text { UK (E) } \\
\text { UK (E) }\end{array}$ & $\begin{array}{l}3 \\
5 \mathrm{D} \\
8 \\
14\end{array}$ & $\begin{array}{l}42 \\
42 \\
42 \\
42\end{array}$ \\
\hline $\begin{array}{l}\text { LC810A } \\
\text { LC1047D }\end{array}$ & $\begin{array}{l}6 \\
1\end{array}$ & $\begin{array}{l}\text { UK (E) } \\
\text { UK (E) }\end{array}$ & $\begin{array}{l}1 \\
5\end{array}$ & $\begin{array}{l}45 \\
45\end{array}$ \\
\hline
\end{tabular}

* Representative strains : all representative strains were apparently unrelated to any other representative strain.

$\uparrow$ Similar strains: the number of strains (including the representative strain) found to have the same RFLP pattern.

¥ Source: the country of origin (environmental strains) or country in which Legionnaires' disease was thought to be contracted (clinical strains). C, clinical strains; E, environmental isolates.

$\S$ Serogroup: as defined by Harrison and Taylor. ${ }^{4}$ Note 5D indicates the strain reacts like a Dallas-1E strain rather than a Cambridge-2 strain.

$\|$ RFLP type: the number allocated to a particular pattern of bands obtained with $\mathrm{NciI}$ and $\lambda 2021 .^{2}$ 


\section{Results}

\section{Relationship of RFLP type to serogroup}

The standard RFLP typing scheme was used to examine more than 500 strains of $L$. pneumophila; 51 RFLP types were identified. Of these, 19 types comprised strains of more than one serogroup (table II). Several RFLP types $(5,26$ and 28$)$ included strains of five or more serogroups. These data confirm and extend our earlier observations that RFLP type shows little concordance with serogroup. ${ }^{3}$

\section{Extended restriction fragment analysis of selected strains}

To determine if sharing the same RFLP type indicates that strains are genotypically indistinguishable or merely that they are superficially similar, three groups of strains were selected for analysis (table I). Restriction endonuclease digests of the strains were prepared with the restriction enzymes listed earlier. To enable comparison of even minor differences, all strains in a particular group were electrophoresed on the same agarose gel. The DNA fragments generated were analysed either directly after ethidium bromide staining (REA) or after Southern blotting and hybridisation with one of the probes listed above. The results obtained were as described below.

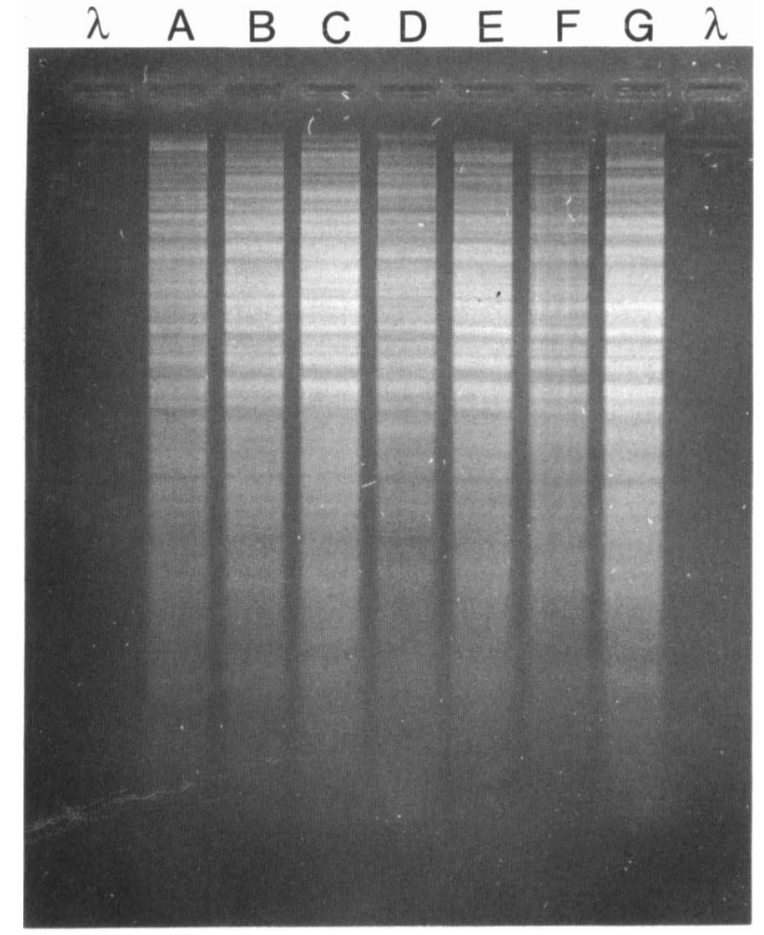

Fig. 2. Ncil restriction fragments from the seven strains representative of RFLP type 28 (table I group i) stained with ethidium bromide $0.5 \mu \mathrm{g} / \mathrm{ml}$ for $30 \mathrm{~min}$. Tracks marked $\lambda$ show a mixture of EcoRI- and PstI-digested $\lambda$ phage DNA. Track A, ML68; B, LC727A;C, LC232; D, LC100; E, LC1; F, LC802; G, NCTC12000.

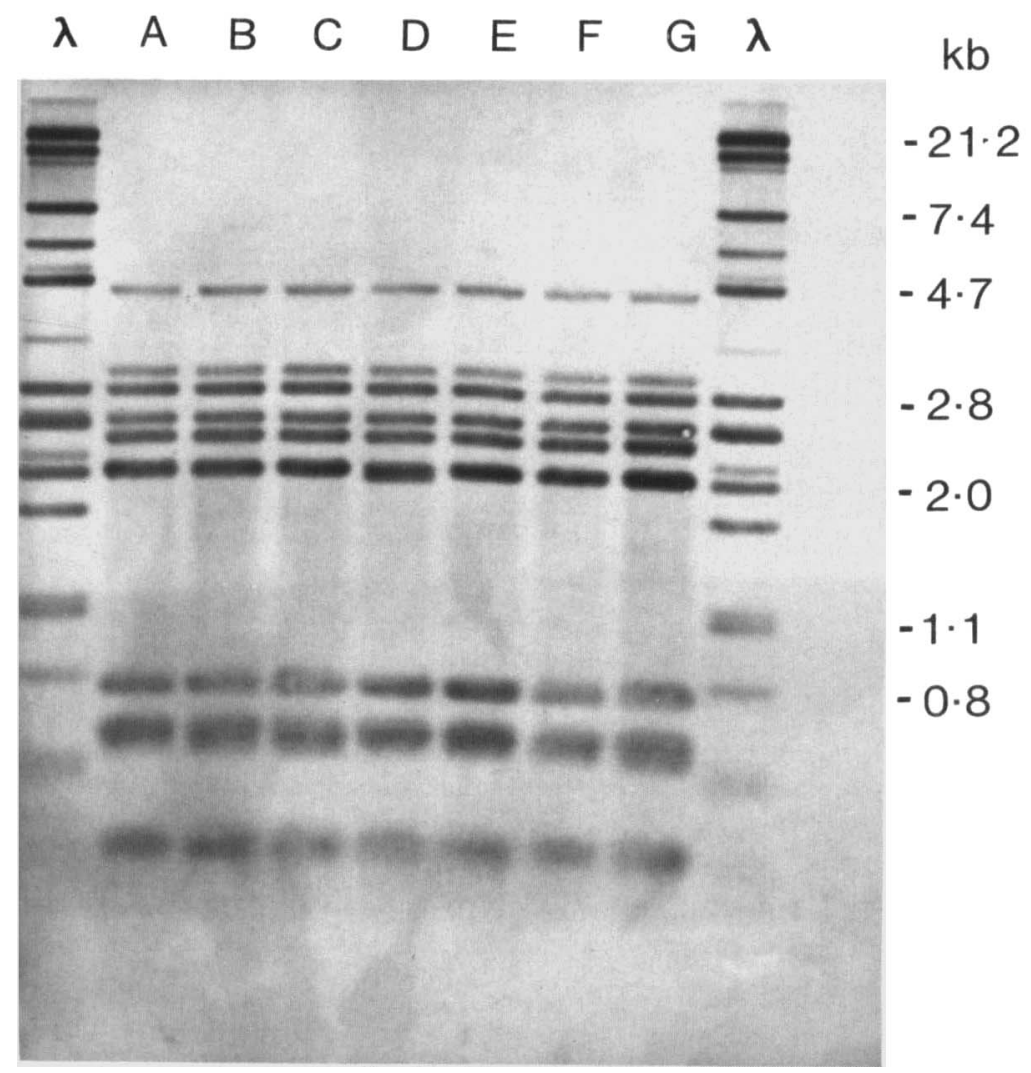

Fig. 1. NciI restriction fragments from the seven strains representative of RFLP type 28 (table I group i) detected with $\lambda 142$. Tracks marked $\lambda$ show a mixture of EcoRI- and PstI-digested $\lambda$ phage DNA. Track A, ML68; B, LC727A; C, LC232; D, LC100; E, LC1; F, LC802; G, NCTC 12000. 
Table III. Restriction fragment patterns obtained for strains in groups (ii) and (iii) (table I) with each enzyme-probe combination*

\begin{tabular}{|c|c|c|c|c|c|c|c|c|c|c|c|c|}
\hline \multirow{3}{*}{ Group } & \multirow{3}{*}{$\begin{array}{c}\text { Strain } \\
\text { no. }\end{array}$} & \multirow{3}{*}{$\begin{array}{l}\text { Sero- } \\
\text { group }\end{array}$} & \multicolumn{10}{|c|}{ Enzyme-probe combination } \\
\hline & & & \multicolumn{4}{|c|}{ NciI } & \multicolumn{2}{|c|}{ Avall } & \multicolumn{2}{|c|}{ BanI } & \multicolumn{2}{|c|}{ HaeIII } \\
\hline & & & REA $\ddagger$ & $\lambda 2021$ & $\lambda 142$ & Leg1 & REA & $\lambda 142$ & REA & $\lambda 2021$ & REA & $\lambda 2021$ \\
\hline \multirow[t]{4}{*}{ (ii) } & $\begin{array}{l}\text { ML54 } \\
\text { LC563 }\end{array}$ & $\begin{array}{l}1 \\
3\end{array}$ & $\begin{array}{l}\mathrm{A} 1 \\
\mathrm{~A} 1\end{array}$ & $\begin{array}{l}26 \\
26\end{array}$ & $\begin{array}{l}\text { B1 } \\
\text { B1 }\end{array}$ & $\begin{array}{l}\mathrm{C} 1 \\
\mathrm{C} 2\end{array}$ & $\begin{array}{l}\text { D1 } \\
\text { D1 }\end{array}$ & $\begin{array}{l}\ldots \\
\cdots\end{array}$ & $\begin{array}{l}\mathrm{F} 1 \\
\mathrm{Fl}\end{array}$ & $\begin{array}{l}\text { G1 } \\
\text { G1 }\end{array}$ & $\begin{array}{l}\mathrm{H} 1 \\
\mathrm{H} 1\end{array}$ & $\begin{array}{l}\text { Il } \\
\text { I1 }\end{array}$ \\
\hline & $\left.\begin{array}{l}\text { LC362 } \\
\text { LC366 }\end{array}\right\}$ & $\begin{array}{l}6 \\
6\end{array}$ & $\begin{array}{l}\mathrm{A} 1 \\
\mathrm{Al}\end{array}$ & $\begin{array}{l}26 \\
26\end{array}$ & $\begin{array}{l}\text { B1 } \\
\text { B1 }\end{array}$ & $\begin{array}{l}\mathrm{Cl} \\
\mathrm{Cl}\end{array}$ & $\begin{array}{l}\text { NT } \\
\text { NT }\end{array}$ & $\begin{array}{l}\cdots \\
\cdots\end{array}$ & $\begin{array}{l}\mathrm{F} 1 \\
\mathrm{~F} 1\end{array}$ & $\begin{array}{l}\text { G1 } \\
\text { G1 }\end{array}$ & $\begin{array}{l}\mathrm{H} 1 \\
\mathrm{H} 1\end{array}$ & $\begin{array}{l}\text { I1 } \\
\text { I1 }\end{array}$ \\
\hline & $\left.\begin{array}{l}\text { LRU57 } \\
\text { LC202 } \\
\text { LC443 } \\
\text { LC207 } \\
\text { LC441 } \\
\text { LC204 } \\
\text { LC442 }\end{array}\right\}$ & $\begin{array}{r}6 \\
6 \\
6 \\
6 \\
12 \\
12 \\
12\end{array}$ & $\begin{array}{l}\text { A1 } \\
\text { A1 } \\
\text { A1 } \\
\text { A1 } \\
\text { A1 } \\
\text { Al } \\
\text { Al }\end{array}$ & $\begin{array}{l}26 \\
26 \\
26 \\
26 \\
26 \\
26 \\
26\end{array}$ & $\begin{array}{l}\text { B1 } \\
\text { B1 } \\
\text { B1 } \\
\text { B1 } \\
\text { B1 } \\
\text { B1 } \\
\text { B1 }\end{array}$ & $\begin{array}{l}\mathrm{Cl} \\
\mathrm{Cl} \\
\mathrm{Cl} \\
\mathrm{Cl} \\
\mathrm{Cl} \\
\mathrm{Cl} \\
\mathrm{Cl}\end{array}$ & $\begin{array}{l}\text { NT } \\
\text { NT } \\
\text { NT } \\
\text { NT } \\
\text { NT } \\
\text { NT } \\
\text { NT }\end{array}$ & $\begin{array}{l}\cdots \\
\cdots \\
\cdots \\
\cdots \\
\cdots \\
\cdots \\
\cdots\end{array}$ & $\begin{array}{l}\text { F1 } \\
\text { F1 } \\
\text { F1 } \\
\text { F1 } \\
\text { F1 } \\
\text { F1 } \\
\text { F1 }\end{array}$ & $\begin{array}{l}\text { G1 } \\
\text { G1 } \\
\text { G1 } \\
\text { G1 } \\
\text { G1 } \\
\text { G1 } \\
\text { G1 }\end{array}$ & $\begin{array}{l}\text { H1 } \\
\text { H1 } \\
\text { H1 } \\
\text { H1 } \\
\text { H1 } \\
\text { H1 } \\
\text { H1 }\end{array}$ & $\begin{array}{l}\text { I1 } \\
\text { I1 } \\
\text { I1 } \\
\text { I1 } \\
\text { I1 } \\
\text { I1 } \\
\text { I1 }\end{array}$ \\
\hline & LC467 & 10 & $\mathrm{~A} 1$ & 26 & B1 & $\mathrm{Cl}$ & D1 & $\ldots$ & F1 & G1 & H1 & I1 \\
\hline (iii) & $\left.\begin{array}{l}\text { LC390 } \\
\text { LC392 } \\
\text { LC393 } \\
\text { LC394 } \\
\text { LC396 } \\
\text { LC398 } \\
\text { LC395 } \\
\text { LC397 } \\
\text { LC384 } \\
\text { LC385 } \\
\text { LC389 } \\
\text { LC391 }\end{array}\right\}$ & $\begin{array}{r}6 \\
6 \\
6 \\
6 \\
6 \\
6 \\
10 \\
10 \\
10 \\
10 \\
10 \\
10\end{array}$ & $\begin{array}{l}\text { A2 } \\
\text { A2 } \\
\text { A2 } \\
\text { A2 } \\
\text { A2 } \\
\text { A2 } \\
\text { A3 } \\
\text { A3 } \\
\text { A3 } \\
\text { A3 } \\
\text { A3 } \\
\text { A3 }\end{array}$ & $\begin{array}{l}28 \\
28 \\
28 \\
28 \\
15 \\
15 \\
15 \\
15 \\
15 \\
15 \\
15 \\
15\end{array}$ & $\begin{array}{l}\text { B2 } \\
\text { B2 } \\
\text { B2 } \\
\text { B2 } \\
\text { B2 } \\
\text { B2 } \\
\text { B3 } \\
\text { B3 } \\
\text { B3 } \\
\text { B3 } \\
\text { B3 } \\
\text { B3 }\end{array}$ & $\begin{array}{l}\ldots \\
\ldots \\
\ldots \\
\ldots \\
\ldots \\
\ldots \\
\ldots \\
\cdots \\
\cdots \\
\cdots \\
\cdots \\
\cdots\end{array}$ & $\begin{array}{l}\text { D2 } \\
\text { D2 } \\
\text { D2 } \\
\text { D2 } \\
\text { D2 } \\
\text { D2 } \\
\text { D3 } \\
\text { D3 } \\
\text { D3 } \\
\text { D3 } \\
\text { D3 } \\
\text { D3 }\end{array}$ & $\begin{array}{l}\text { E1 } \\
\text { E1 } \\
\text { E1 } \\
\text { E1 } \\
\text { E1 } \\
\text { E1 } \\
\text { E2 } \\
\text { E2 } \\
\text { E2 } \\
\text { E2 } \\
\text { E2 } \\
\text { E2 }\end{array}$ & $\begin{array}{l}\ldots \\
\cdots \\
\cdots \\
\cdots \\
\cdots \\
\cdots \\
\cdots \\
\cdots \\
\cdots \\
\cdots \\
\cdots \\
\cdots\end{array}$ & $\begin{array}{l}\ldots \\
\ldots \\
\ldots \\
\ldots \\
\ldots \\
\ldots \\
\ldots \\
\ldots \\
\ldots \\
\ldots \\
\ldots \\
\ldots\end{array}$ & $\begin{array}{l}\ldots \\
\cdots \\
\ldots \\
\ldots \\
\cdots \\
\cdots \\
\ldots \\
\cdots \\
\cdots \\
\cdots \\
\cdots \\
\cdots\end{array}$ & $\begin{array}{l}\ldots \\
\ldots \\
\cdots \\
\ldots \\
\ldots \\
\cdots \\
\cdots \\
\cdots \\
\cdots \\
\cdots \\
\cdots \\
\cdots\end{array}$ \\
\hline
\end{tabular}

* StyI results are not shown (see text for details).

$\uparrow$ Strains bracketed together are epidemiologically associated as described in the text; LC389 and LC390 were both isolated from the same patient.

$\ddagger$ REA, total restriction endonuclease digest (unprobed).

$\ldots$, Not determined.

NT, not typable (the strain was resistant to digestion with this enzyme).

(i) When the seven representative strains of RFLP type 28 (table I) were examined with $\mathrm{NciI}$ and probe $\lambda 142$, the patterns obtained were indistinguishable (fig. 1). However, each of the strains were distinguishable when examined by REA, although the profiles were very similar, differing only in a few high-mol.-wt bands (fig. 2). Examination of the four L. pneumophila serogroup 1 RFLP type 28 strains (table II) by REA with $\mathrm{NciI}$ revealed that these also were distinct from each other. Thus, each of the 11 RFLP type 28 strains examined, although very similar, was of a distinguishable genotype.

(ii) Results obtained from the analyses of the 12 RFLP type 26 strains are shown in table III. Following $\mathrm{Nci}$ I digestion all strains gave indistinguishable patterns by REA and when probed with either $\lambda 20 / 21$ or $\lambda 142$, but a minor shift in the position of one band was seen for LC563, compared to the 11 other strains, when probe $\lambda$ LEGl was used. Nine of the 12 strains were not digested by AvaII; REA profiles of the other three were indistinguishable. When the strains were digested with StyI, clear RFLP patterns could not be identified as there were reproducible differences in the degree of digestion observed for different strains. It seems likely that this was due to the modification (e.g., by methylation) of StyI sites to different extents in different strains.

The strains were also indistinguishable following HaeIII digestion both by REA and RFLP typing with $220 / 21$. However, it should be noted that HaeIII generated a large number of small restriction fragments and, consequently, minor differences were hard to detect. RFLP typing with $\lambda 20 / 21$ and BanI revealed indistinguishable patterns for all strains. Thus, all strains were very similar and no restriction enzymeprobe combination revealed even minor differences between the strains of serogroups 6 and 12 .

(iii) The results obtained from the analysis of the Belgian hospital strains are shown in table III. With the combination of $N c i$ and $\lambda 20 / 21$, the serogroup 10 strains and environmental serogroup 6 strains were confirmed as RFLP type 15, and the clinical serogroup 6 strains as RFLP type 28 (fig. 3). However, REA with the same $\mathrm{Ncil}$ digests distinguished these strains into two very similar groups corresponding to their serogroups; the environmental serogroup 6 strains were now indistinguishable from the clinical serogroup 6 strains but distinguishable from the serogroup 10 strains. This was confirmed by REA with AvaII, and RFLP typing with $N c i \mathrm{I}, \lambda 142$ and $A v a I I, \lambda 142$. All 12 


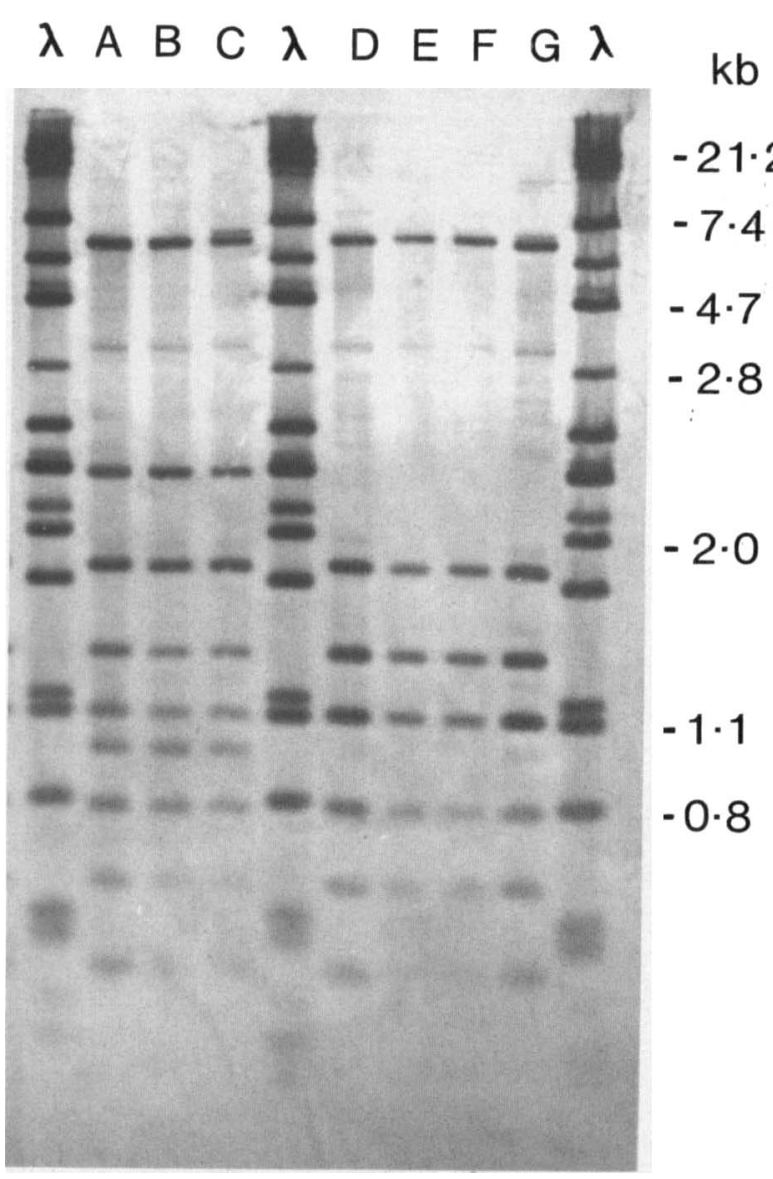

Fig. 3. $N c i$ restriction fragments from the Belgian hospital strains detected with $\lambda 20 / 21$. Tracks marked $\lambda$ show a mixture of EcoRIand PstI-digested $\lambda$ phage DNA. Track A, LC390; B, LC392; C, LC394; D, LC396; E, LC398; F, LC395; G, LC384.

strains were resistant to digestion with StyI. Thus, three very similar genotypes (A2/28, A2/15, A3/15) were identified in this group of strains.

\section{Discussion}

In 1985 , Selander et al. ${ }^{9}$ undertook a study of the genetic structure of $L$. pneumophila populations by electrophoretic analysis of protein polymorphisms of enzymes encoded by 22 structural genes. This work assumed that evolutionary convergence to the same multilocus genotype is highly improbable and, thus, enzyme variants can be used as markers to estimate genetic relatedness. In their study, 62 distinct electrophoretic types (ETs) were identified and the authors concluded that the species $L$. pneumophila is highly polymorphic and clonal in population. They also noted that the division of strains into serogroups had little relationship to the underlying genetic structure. These conclusions, based on an indirect measure of genetic diversity, were later confirmed by van Ketel $^{1}$ who attempted to determine genotypic differences directly by comparison of REA profiles. He examined strains from seven environmental sources that contained strains of more than one serogroup and found that, for each of six sources, the REA profiles of the strains they contained were very similar.

There are several limitations to the use of REA in this context. These include: (i) REA profiles can be compared with confidence only when the digests are run on the same gel, hence it is very difficult to compare large numbers of strains; (ii) it is very difficult to identify small differences between REA profiles, thus the similarity in profiles may be over-estimated; and (iii) it is possible that differences in REA profiles may occur because of differences in plasmid not chromosomal DNA. ${ }^{1{ }^{10}}$ This study attempted to overcome these problems by using RFLP typing in addition to REA. The standard RFLP-typing scheme used here (NciI, 220/21) has been thoroughly evaluated previously. ${ }^{2}$ It is highly discriminatory (at least 51 RFLP types), reproducible, and the patterns obtained are not affected by plasmid-derived DNA. Although only a small fraction of the total genome is compared by RFLP typing, the inclusion of other probe-restriction enzyme combinations significantly increases this proportion and thus improves the discrimination of the method. $^{8}$

The data presented here confirm and extend the earlier conclusions $\mathbf{s}^{\mathbf{1 , 3}, 9}$ although differences are apparent. Selander et al. ${ }^{9}$ reported that 6 of 62 ETs comprised strains of more than one serogroup, whereas we found this was the case for 19 of 51 RFLP types. This apparent difference in findings is possibly because only 17 of the 62 ETs comprised more than a single strain, in contrast to 27 of the 51 RFLP types. Alternatively it may show that results of serogrouping and ET typing correspond more closely than do those of serogrouping and RFLP typing.

It was not the intention of this study to compare protein electromorph (ET typing) data with RFLP data; however, coincidentally a few strains examined here were also examined by Selander et al. ${ }^{9}$ It is noteworthy that the only two strains of the same ET to be examined here were clearly distinguishable by RFLP typing. Selander $e t$ al. reported that the strains Leiden-1E (NCTC 12000) and Cambridge-2 (NCTC 11417) were both ET 31, but we found that they were of very dissimilar RFLP types (only five bands in common with four and nine unique bands for RFLP types 28 and 50 respectively). Thus, in this instance, in contradiction to the supposition of Selander et al., it seems probable that convergence of distinct strains to the same multilocus genotype has occurred.

To determine if sharing the same RFLP type indicates that strains are genotypically indistinguishable or merely that they are superficially similar, three groups of strains were selected for detailed analysis. Group (i) represented strains of the RFLP type showing most serological diversity so far identified (RFLP type 28). This comprised seven strains each of a different serogroup. Minor but clear differences were seen between all these strains by REA. This prompted examination for the four serogroup 1 RFLP type 28 strains, and these also were all distinguishable by 
REA. Although the possibility that some of the differences were due to plasmid-derived DNA cannot be excluded, this appears unlikely because only one of the four serogroup 1 strains carried a plasmid (data not shown). Alternative possibilities are (1) that transposons or lysogenic phage were present within the genomes of the strains at differing sites or (2) the RFLP type 28 represents a well established clone that has diverged genotypically to give many similar subtypes.

The second group of strains (RFLP type 26) showed a very high degree of similarity, being indistinguishable by REA and RFLP typing with any of the restriction enzymes or probes used. The serogroup 6 and serogroup 12 strains could be distinguished from the serogroup 1, serogroup 3 and serogroup 10 strains solely by the phenotypic characteristic of susceptibility or resistance to Avall digestion. The failure to distinguish between the clinical and environmental serogroup 6 and serogroup 12 strains from the LD outbreak in a renal unit (table III (ii) LRU57-LC442) strongly suggests that these strains are derived from a single clone which is able to express different serogroup antigens.

The third group comprised serogroup 6 and serogroup 10 strains isolated from patients and environmental sites in a Belgian hospital. Previous work ${ }^{3}$ had shown that the serogroup 10 strains (environmental and clinical) and environmental serogroup 6 strains were all RFLP type 15, but the clinical serogroup 6 strains were RFLP type 28 . In this study, REA clearly differentiated the strains in concordance with sero- grouping, as did RFLP typing with AvaII. However, the two REA profiles identified were very similar, as were the RFLP type 15 and type 28 patterns (fig. 3), suggesting that the strains are genotypically closely related. Furthermore, there were minor differences in the RFLP profiles (with both AvaII and NciI) of the two serogroup 6 RFLP type 15 strains compared to the serogroup 10 RFLP type 15 strains. However, these differences are not easily discernible and not significant enough to be considered in determining the RFLP type. Therefore, it is possible that the strains represent a single diverging clone and that the genotype A2/15 (see table III) is an intermediate between $A 2 / 28$ and $A 3 / 15$, the change in antigenic expression accompanying the clonal divergence.

Taken together, the data from the two components of the study strongly suggest that RFLP type correlates with genotype. In some cases, strains of a particular RFLP type are indistinguishable genotypically whereas in other instances they are very similar but appear to be diverging. In either case, RFLP and REA analysis give a more accurate indication of the ancestral relationship between one strain and another than does serogrouping. For this reason, when the interactions of legionella strains are being monitored over a long timespan or when two or more strains are being compared (e.g., in virulence studies), the RFLP typing or REA should be employed, as the reliance on serological markers could be misleading.

We are grateful to all our microbiologist colleagues, particularly Drs J. Barbaree, H. Ezzeddine and T. Makin, who provided strains for us to examine.

\section{References}

1. van Ketel RJ. Similar DNA restriction endonuclease profiles in strains of Legionella pneumophila from different serogroups. J Clin Microbiol 1988; 26: 1838-1841.

2. Saunders NA, Harrison TG, Haththotuwa A, Kachwalla N, Taylor AG. A method for typing strains of Legionella pneumophila serogroup 1 by analysis of restriction fragment length polymorphisms. $J$ Med Microbiol 1990; 31: 45-55.

3. Harrison TG, Saunders NA, Haththotuwa A, Doshi N, Taylor AG. Typing of Legionella pneumophila serogroups 2-14 strains by analysis of restriction fragment length polymorphisms. Lett Appl Microbiol 1990; 11: 189-192.

4. Harrison TG, Taylor AG (eds). A laboratory manual for Legionella. Chichester, John Wiley and Sons. 1988.

5. Meigh RE, Makin T, Scott MH, Hart CA. Legionella pneumophila serogroup 12 pneumonia in a renal transplant recipient: case report and environmental observations. $J$ Hosp Infect 1989; 13: 315-319.

6. Ezzeddine H, van Ossel C, Delmee M, Wauters G. Legionella spp. in a hospital hot water system: effect of control measures. J Hosp Infect 1989; 13: 121-131.

7. Saunders NA. Analysis of restriction fragment length polymorphisms (RFLPs) for epidemiological tracing of bacteria using non-radioactive probes. Focus $1989 ; 11$ : 47-49.

8. Saunders NA, Harrison TG, Haththotuwa A, Taylor AG. A comparison of probes for restriction fragment length polymorphism (RFLP) typing of Legionella pneumophila serogroup 1 strains. J Med Microbiol 1991; 35: 152-158.

9. Selander RK, McKinney RM, Whittam TS et al. Genetic structure of populations of Legionella pneumophila. $J$ Bacteriol 1985; 163: 1021-1037.

10. Harrison TG, Saunders NA, Haththotuwa A, Hallas G, Birtles RJ, Taylor AG. Phenotypic variation amongst genotypically homogeneous Legionella pneumophila serogroup 1 isolates: implications for the investigation of outbreaks of Legionnaires' disease. Epidemiol Infect 1990; 104: 171-180. 